\title{
TWO-DIMENSIONAL COMPUTER MODELLING OF POLYCRYSTALLINE FILM GROWTH
}

\author{
A.J. DAMMERS AND S. RADELAAR \\ DIMES / Section Submicron Technology, \\ Laboratory of Applied Physics, Delft University of Technology, \\ P.O. Box 5046, 2600 GA Delft, the Netherlands
}

\begin{abstract}
Polycrystalline thin films, deposited from a vapour phase, often show a columnar morphology. We present computer simulations of a 2D model of the polycrystalline growth process. The model consists of randomly oriented squares, growing from a line. We find, that the characteristic length scale $\langle\Delta x\rangle$ of the growing surface (average edge length projected on the substrate) diverges as a function of time according to a power law $\langle\Delta x>\sim t p$, with $p \approx 0.52$.
\end{abstract}

\section{INTRODUCTION}

Thin solid films, grown from a vapour phase with techniques such as sputtering, evaporation or chemical vapour deposition, often have a columnar morphology. The intrinsic structure of these columns may be amorphous or crystalline. This depends on the process conditions (substrate temperature, gas pressure etc.) and essentially it relates to the mobility of the adatoms on the surface during deposition. 1,2

In the low mobility regime amorphous layers are formed. Computer simulations of atomistic (ballistic deposition) 3,4 and continuum models $5,6,7,8$ have given a great deal of insight into the factors governing this growth process. Major physical concepts (relevant to e.g. evaporation or sputter deposition) can be roughly summarized as follows. A surface element collects an amount of material, which depends on its 
orientation relative to the incoming flux. Atoms are assumed to have a mean free path, which is larger than typical height differences of the substrate, so protruding parts of the surface collect material at the expense of regions further down the vapour stream. On the other hand, the effect of this nonuniform deposition is counteracted by redistribution of the material over the surface, which may vary from local restructuring to long range diffusion. The net result will be, at moderate degrees of atomic mobility, formation of columns, originating from favourably located surface regions.

If the deposited material has sufficiently high mobility, the shadowing phenomenon described above will no longer be adequate to cause texture formation. However, now another mechanism appears to become active. Crystals nucleate on the substrate and grow freely until impingement. Subsequently, these growing domains compete for survival and columns are formed. Due to the intrinsic growth anisotropy (facets) of the crystals, crystallographic texture appears to develop. Crystals with their fastest growing directions perpendicular to the substrate grow at the expense of less favourably oriented ones. This "principle of evolutionary selection" was proposed by Van der Drift ${ }^{9}$ in order to explain preferential crystallographic orientation, as observed experimentally for numerous systems.

In order to gain more insight into the morphology of columnar polycrystalline layers, we developed a $2 \mathrm{D}$ computer model simulating the growth of such films. Clearly, it has its limitations compared to a true 3D approach, but we will show that even such a simple model has non-trivial properties. In particular, the long time behaviour of the (2D) growth process appears to be qualitatively similar to (3D) experimental data. 10 Moreover, our results for polycrystalline layers are comparable to recently obtained behaviour of growing amorphous layers. 7

\section{Simulations}

The 2D model which we study in this paper consists of a line of length $L$, on which randomly oriented equidistant square crystals (spacing d) nucleate simultaneously (Fig. 1). The growth rate (thickness of the layer deposited per second) of all edges is $g$. As a convenient unit of time $t$ we use $\{d / g\}$. In order to minimize finite size effects, periodic boundary conditions are applied. In the statistical analysis of the simulations we focus on surface features. A top view of the layer yields the projected edge length $\Delta x$ of the surviving crystals as a characteristic length scale of the surface. 
Details of the computer implementation of this model were reported previously. 11

Deposition Flux
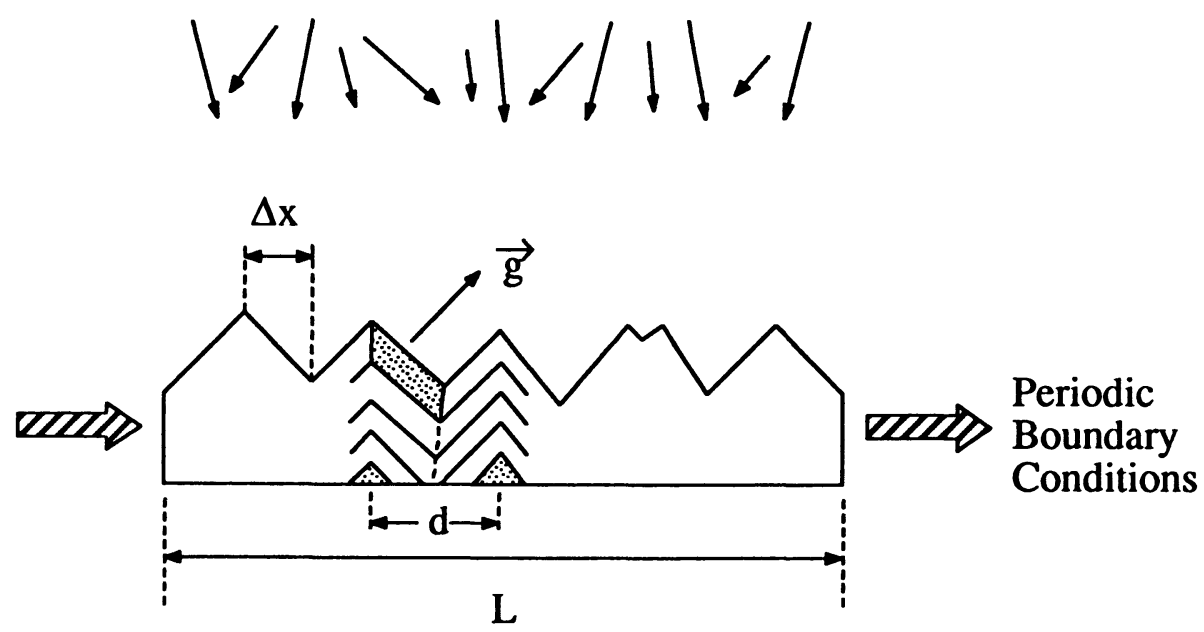

Figure 1. Model system studied in this paper. A line of length $L$ is initially covered with randomly oriented crystal nuclei (squares) positioned at regular intervals (spacing $d$ ). All crystal edges grow with the same rate $g$. Periodic boundary conditions are employed. $\Delta x$ is the length of an edge, projected on the substrate.

The result of a typical simulation is given in Fig. 2. Essentially this is the classical example of Van der Drift ${ }^{9}$. Clearly one observes the "principle of evolutionary selection". Crystals with a direction of fastest growth more or less perpendicular to the substrate grow at the expense of less favourably oriented ones. This leads to the well known columnar structure with crystallographic texture. 


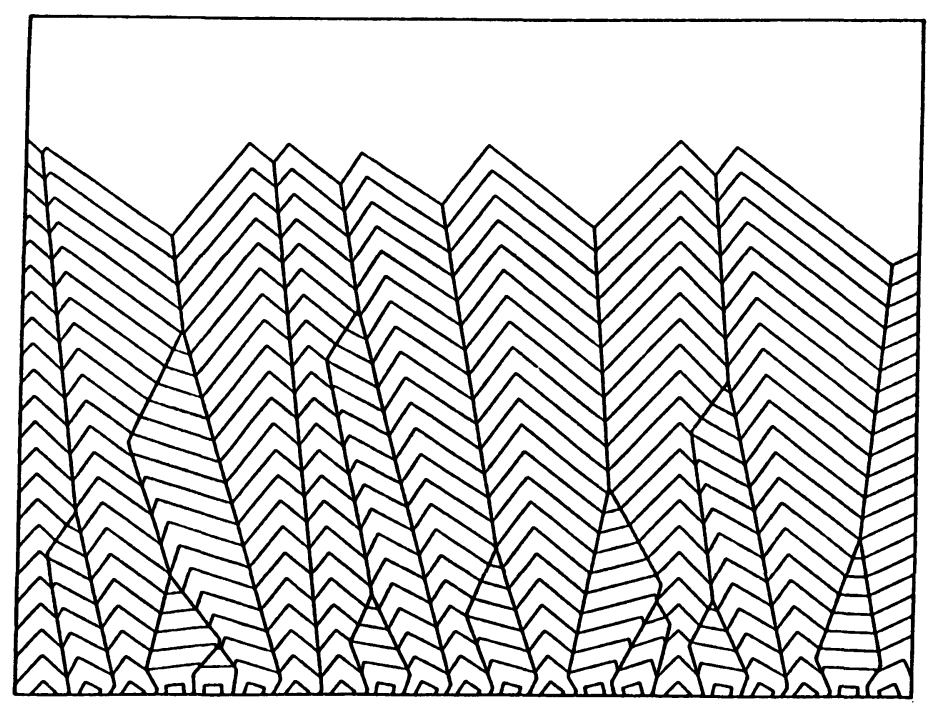

Figure 2. Growth of randomly oriented squares from a line. The squares nucleate simultaneously and grow at equal rates. This is a detail of a larger simulation, so periodic boundary conditions are not visible.

From Fig. 2 one may get the impression that after an initial period the surface profile reaches a steady state. However, the coarsening process continues. This phenomenon can be formulated in a more quantitative way, by looking at the behaviour of the scaled average projected edge length $\langle\Delta x\rangle / L$ with time \#. In Fig. 3 we present $\log (\langle\Delta x\rangle / L)$ as a function of $\log (t)$ for a system of 5000 squares, as obtained by averaging the results of 4 simulations. Clearly, a linear relationship exists, which implies a power law

$$
<\Delta x>\sim t^{p}
$$

with $p \approx 0.52$.

\footnotetext{
\# We use the scaled (dimensionless) quantity $\langle\Delta\rangle / L=$ (nr. of edges) $^{-1}$
} 


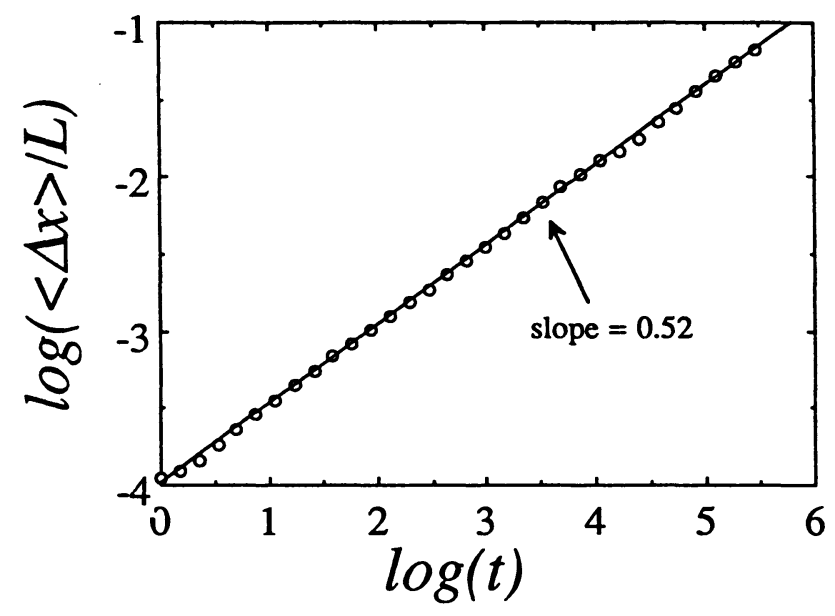

Figure 3. The (scaled) average projected edge length $<\Delta x>/ L$ as a function of time $t$, given in units of $\{d / g\}$. The initial configuration consists of 5000 equidistant, randomly oriented, squares. This graph was obtained by averaging over 4 runs (different sets of random numbers).

\section{Discussion}

The results of the simulations presented here unifiy polycrystalline film growth with evolution of surfaces of amorphous layers. Tang et al. 7 reduced a continuum model for growth of amorphous layers 5,6 to the evolution of isotropic wave fronts, originating from the initial surface. Essentially, the columns are then determined by the waves related to (local) maxima of the surface profile. In other words, the long-time behaviour of the surface is indistinguishable from that of one, which originates from isotropically growing isolated domains, although the real initial surface is continuous. Thus, the main difference between our polycrystalline model and the amorphous one cited above is the anisotropy of the growth rates, as reflected in the presence of crystal facets.

This qualitative similarity between evolution of amorphous and polycrystalline layers is enhanced by our quantitative results. Tang et al. 7 also found a power law like (1) for 2D as well as 3D amorphous model systems. The exponent $p$ could be tuned from 0.2 to 0.9 by specifying the 
initial height distribution and moreover by adding noise. Some (3D) results appeared to agree with power law behaviour of sputtered amorphous SiC films, as observed experimentally by Roy and Messier ${ }^{10}$. In the latter paper also a power law was reported for polycrystalline $\mathrm{SiC}$ films. This indicates that our simulations relate to essential features of evolution of polycrystalline surfaces, but as we only studied a 2D model system, statements about quantitative agreement would be premature.

Further simulations on systems with various crystal shapes and distributions of initial sizes, positions and orientations are in progress. On the other hand, it would be quite interesting to analyse more experimental data, in particular on materials other than $\mathrm{SiC}$, in order to assess the universality of power law behaviour for growing polycrystalline columnar thin films.

\section{REFERENCES}

1. B.A. Movchan and A.V. Demchishin, Phys. Met. Metallogr. USSR 28, 83 (1969).

2. J.A. Thornton, Ann. Rev. Mater. Sci. 7, 239 (1977).

3. H.J. Leamy, G.H. Gilmer and A.G. Dirks, in: Current Topics in Materials Science, Vol.6, E. Kaldis (Ed.), North Holland, Amsterdam (1980), pp. 309-344.

4. P. Meakin, CRC Critical Reviews in Solid State and Materials Science 13, 143 (1986).

5. M. Kardar, G. Parisi and Y. Zhang, Phys. Rev. Lett. 56, 889 (1986).

6. G.S. Bales and A. Zangwill, Phys. Rev. Lett. 63, 692 (1989).

7. C. Tang, S. Alexander and R. Bruinsma, Phys. Rev. Lett. 64, 772 (1990).

8. G.S. Bales, R. Bruinsma, E.A. Eklund, R.P.U. Karunasiri, J. Rudnick and A. Zangwill, Science 249, 264 (1990), and references therein.

9. A. van der Drift, Philips Res. Repts. 22, 267 (1967).

10. R.A. Roy and R. Messier, Mat. Res. Soc. Symp. Proc. 38, 363 (1985).

11. A.J. Dammers and S. Radelaar, In: Semiconductor Silicon 1990 [ Proceedings of the Sixth International Symposium on Silicon Materials Science and Technology, Montreal May 6-10, 1990 ], H.G. Huff, K.G. Barraclough and J. Chikawa (Eds.), pp. 813-820. 